\title{
Improving Formate and Methanol Fuels: Catalytic Activity of Single Pd Coated Carbon Nanotubes
}

\author{
Xiuting Li, ${ }^{\dagger, \S}$ Hannah Hodson, ${ }^{\dagger, \S}$ Christopher Batchelor-McAuley, ${ }^{\dagger}$ Lidong Shao, ${ }^{\dagger}$
} and Richard G. Compton* ${ }^{\dagger}$

\begin{abstract}
${ }^{\dagger}$ Department of Chemistry, Physical \& Theoretical Chemistry Laboratory, Oxford University, Oxford OX1 3QZ, United Kingdom
${ }^{\ddagger}$ Shanghai Key Laboratory of Materials Protection and Advanced Materials in Electric Power, Shanghai University of Electric Power, 2103 Pingliang Road, Shanghai 200090, People's Republic of China
\end{abstract}

\section{Supporting Information}

ABSTRACT: The oxidations of formate and methanol on nitrogen-doped carbon nanotubes decorated with palladium nanoparticles were studied at both the singlenanotube and ensemble levels. Significant voltammetric differences were seen. Pd oxide formation as a competitive reaction with formate or methanol oxidation is significantly inhibited at high overpotentials under the high mass transport conditions associated with single-particle materials in comparison with that seen with ensembles, where slower diffusion prevails. Higher electro-oxidation efficiency for the organic fuels is achieved.

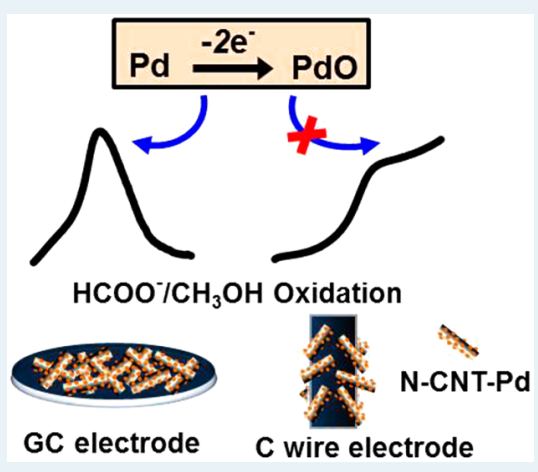

KEYWORDS: formate, methanol, palladium, carbon nanotube, catalytic activity

\section{INTRODUCTION}

Increased interest in alternative fuel sources has led to the separate application of both formate and methanol in fuel cells. Their use as power sources is appealing due to their high energy density and ease of transportation and storage, in contrast to hydrogen fuel cells. ${ }^{1-5}$ Pd-based materials have been intensely studied as superior and cost-effective electrocatalysts in the oxidation of both formate and methanol. ${ }^{6}$ For formate oxidation, it has been suggested that Pd-based catalysts have a better performance in comparison with $\mathrm{Pt}$, as the catalytic reactions on $\mathrm{Pd}$ follow a direct pathway and no poisoning intermediates are produced. ${ }^{7,8}$ Pd-based materials are also considered to be better catalysts in alkaline media for methanol oxidation than Pt-based materials owing to their improved kinetics and, again, lower poisoning from adsorbed intermediates.' However, the instability of the Pd-based catalysts can lead to relatively low energy transformation efficiency. ${ }^{10,11}$ Improving the electrocatalytic stability remains a critical obstacle in the development of Pd-based electrocatalysts in both formate and methanol fuel cells, and a fundamental understanding of $\mathrm{Pd}$ deactivation due to its high surface sensitivity is still incomplete.

The electrocatalytic behavior of newly produced materials are commonly assessed by drop-casting the catalysts onto electrode surfaces and then performing electrochemical tests to analyze their performance. However, the interpretation of such a multilayered, porous drop-cast surface is complex and hard to analyze. $^{13-19}$ Moreover, the most commonly used types of electrodes in these studies are macroelectrodes, at which the mechanism and kinetics can be masked by the limited, slow mass transport conditions.

In this work, we first develop an improved electrode modification method by modifying a carbon microwire electrode in situ with well-separated individual catalyst entities. This modification is achieved by inserting the wire electrode in a very dilute suspension of the nitrogen-doped carbon nanotubes decorated with palladium nanoparticles (N-CNT$\mathrm{Pd})$. Also present in the solution is the redox species of interest (formate or methanol). Over a period of time individual catalyst entities (N-CNT-Pd) continuously and randomly collide with the wire electrode due to their Brownian motion. Some of the N-CNT-Pd becomes immobilized on the electrode surface, forming a layer of low coverage. After immobilization the voltammetric response of the modified electrode is recorded in the same solution. In addition to these surface modification studies, a single-particle electrochemical method based on "nanoimpacts" was applied. This technique relies on the random collision of individual nanoparticles with a potentiostated electrode where control of the potential leads to mediated electron transfer on the surface of the impacting nanoparticle. ${ }^{20-27}$ The current amplification from mediated electron transfer is analyzed and can be applied to the

Received: July 19, 2016

Revised: September 13, 2016

Published: September 14, 2016 

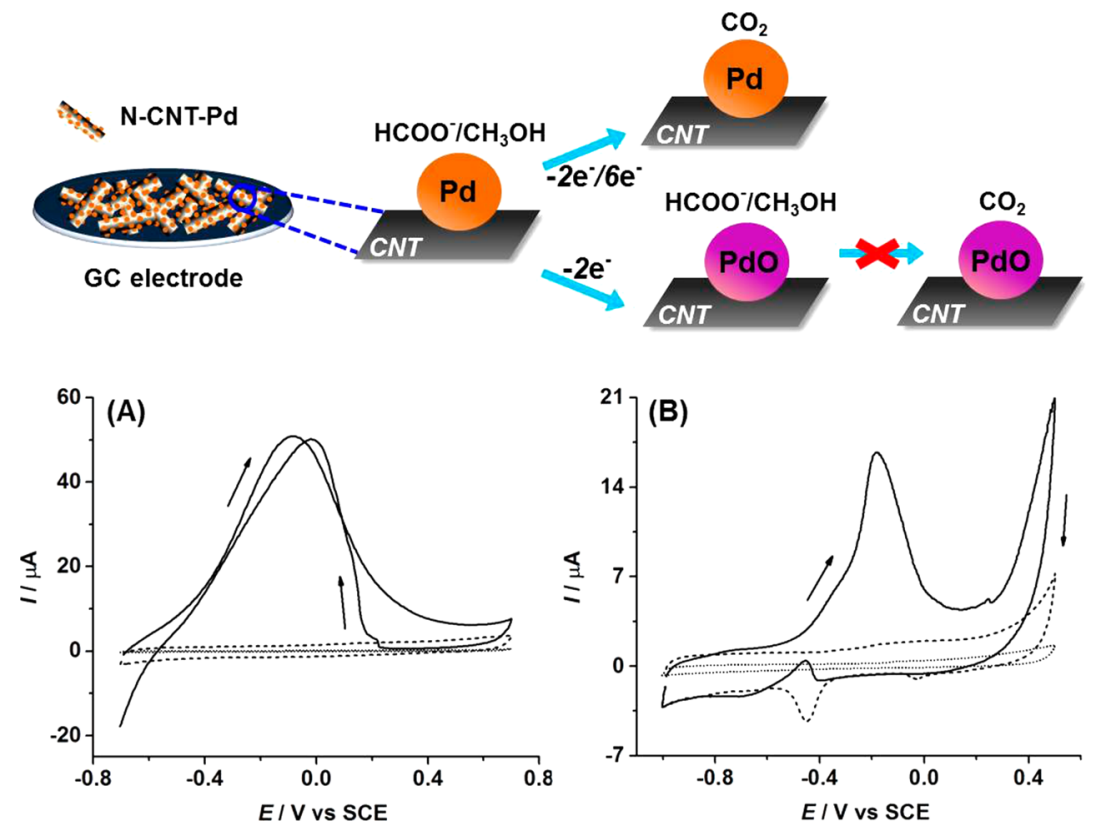

Figure 1. (A) Voltammograms of a GC electrode $(d=3.0 \mathrm{~mm})$ modified with $0.96 \mu \mathrm{g}$ of N-CNT-Pd recorded in $5 \mathrm{M} \mathrm{NaClO}_{4}(\mathrm{aq})$ in the absence (dashed line) and presence (solid line) of $5 \mathrm{M}$ formate. Also shown is the voltammetic response of the bare $\mathrm{GC}^{\text {electrode in } 5 \mathrm{M} \mathrm{NaClO}} 4+5 \mathrm{M}$ formate (dotted line). Scan rate: $50 \mathrm{mV} \mathrm{s}^{-1}$. (B) Voltammograms of a GC electrode $(d=3.0 \mathrm{~mm})$ modified with $1.20 \mu \mathrm{g}$ of N-CNT-Pd in $1 \mathrm{M}$ $\mathrm{NaOH}(\mathrm{aq})$ in the absence (dashed line) and presence (solid line) of $10 \mathrm{M}$ methanol. Also shown is the voltammetric response of the bare GC electrode in $1 \mathrm{M} \mathrm{NaOH}+10 \mathrm{M}$ methanol (dotted line). Scan rate: $50 \mathrm{mV} \mathrm{s}^{-1}$.

fundamental mechanisms and dynamics of electrochemical processes at the single-entity level. ${ }^{28}$ In this work, formate oxidation on individual impacting N-CNT-Pd was studied with the nanoimpact method. The above methods, including both in situ modification and the nanoimpact experiments, lead to the spatial isolation of the catalyst particles on the electrode surface, creating high mass transport conditions for the oxidation processes, which provide new insights into the electrocatalytic mechanisms of formate and methanol oxidation on palladium and how this is influenced by mass transport.

\section{EXPERIMENTAL SECTION}

2.1. Materials. Sodium formate ( $\geq 98 \%)$, methanol (99.99\%), $\mathrm{NaClO}_{4}$, and $\mathrm{NaOH}$ were received from SigmaAldrich. Nitrogen gas $\left(\mathrm{N}_{2}\right)$ was supplied from BOC, Surrey, U.K. All solutions were prepared with deionized water of resistivity not less than $18.2 \mathrm{M} \Omega \mathrm{cm}\left(25^{\circ} \mathrm{C}\right.$, Millipore). The preparation and characterization of nitrogen-doped carbon nanotubes decorated with palladium nanoparticles (N-CNT$\mathrm{Pd})$ were detailed in previous papers. ${ }^{28,29}$

2.2. Cyclic Voltammetry (CV) of Formate and Methanol Oxidation on the GC Electrode Drop-Cast with N-CNT-Pd. A suspension of N-CNT-Pd $\left(9.2 \times 10^{-12} \mathrm{M}\right)$ was prepared by adding $2.4 \mathrm{mg}$ of N-CNT-Pd to $5 \mathrm{~mL}$ of chloroform, followed by sonication in a Fisher Scientific FB15050 ultrasonic bath for $1 \mathrm{~min}$. A glassy-carbon (GC) macroelectrode $(d=3.0 \mathrm{~mm})$ was polished with three grades of diamond spray $(3.0,1.0$, and $0.1 \mu \mathrm{m})$ and then sonicated in water and left to dry under an $\mathrm{N}_{2}$ atmosphere. The as-prepared N-CNT-Pd suspension was then drop-cast on the GC electrode and dried under an $\mathrm{N}_{2}$ atmosphere.

Electrochemical measurements were conducted in a double Faraday cage with an Autolab potentiostat (Metrohm-Autolab BV, The Netherlands) using a three-electrode system at $298 \mathrm{~K}$. For the drop-cast experiments, the modified GC electrode was used as the working electrode, with platinum foil and a saturated calomel electrode (SCE) as the counter electrode and the reference electrode, respectively. Before the measurements a solution containing $5 \mathrm{M}$ formate and $5 \mathrm{M} \mathrm{NaClO}_{4}$ (or $10 \mathrm{M}$ methanol and $1 \mathrm{M} \mathrm{NaOH}$ ) was bubbled with $\mathrm{N}_{2}$ for 10 min to remove dissolved $\mathrm{O}_{2}$, and an atmosphere of $\mathrm{N}_{2}$ was maintained during the experiment. Two control experiments were carried out: an unmodified GC electrode in $5 \mathrm{M}$ formate $+5 \mathrm{M}$ $\mathrm{NaClO}_{4}$ (or $10 \mathrm{M}$ methanol $+1 \mathrm{M} \mathrm{NaOH}$ ) and a modified GC electrode in $5 \mathrm{M} \mathrm{NaClO}_{4}$ (or $1 \mathrm{M} \mathrm{NaOH}$ ).

2.3. CV of Formate and Methanol Oxidation on the Carbon Microwire Electrode in Situ Modified with $\mathrm{N}$ CNT-Pd and the Nanoimpact Experiments. In the in situ modification experiments, the working electrode was carbon fiber microwire electrodes (ca. $7 \mu \mathrm{m}$ in diameter and ca. $1 \mathrm{~mm}$ in length), which were fabricated according to the procedure introduced in our previous work. ${ }^{30}$ Formate or methanol solution was degassed with $\mathrm{N}_{2}$ for $10 \mathrm{~min}$, and then a known concentration of N-CNT-Pd suspension was added and bubbled with $\mathrm{N}_{2}$ for $30 \mathrm{~s}$ to make a well-dispersed and diluted suspension of N-CNT-Pd. CV was recorded immediately after a clean carbon microwire electrode was inserted in the suspension containing formate or methanol and after the electrode was in situ modified in the same solution for a certain time. The nanoimpact experiments were conducted by scanning chronoamperometry immediately after a clean carbon microwire electrode was inserted in the above solutions. For counting and analysis of the current spikes, the software "SignalCounter" was used. ${ }^{31}$

\section{RESULTS AND DISCUSSION}

We first investigate the $\mathrm{CV}$ of formate and methanol oxidation on glassy-carbon (GC) electrodes drop-cast with N-CNT-Pd to evidence the catalytic ability of the N-CNT-Pd toward these oxidation processes. Mechanistic considerations are made. 
Second, we measure and analyze the $\mathrm{CV}$ of first formate and second methanol oxidation on carbon microwire electrodes which have been modified in situ with individual spatially well separated N-CNT-Pd tubes via their random collision and immobilization on the wire electrode. Third, nanoimpact experiments are conducted by recording chronoamperometry at a carbon microwire electrode in an aqueous suspension of $\mathrm{N}$ CNT-Pd in the presence of formate. Current spikes in the chronoamperograms resulting from formate oxidation on individual (single) impacting N-CNT-Pd are detected and analyzed. Similar experiments are attempted for the oxidation of methanol. Finally, the results from all the experiments are compared and chemical mechanisms inferred and discussed.

3.1. CV of Drop-Cast N-CNT-Pds. The cyclic voltammetric responses of formate and methanol oxidation were separately investigated on N-CNT-Pd modified GC electrodes (of ca. 3.0 $\mathrm{mm}$ in diameter). To study the formate oxidation, an unmodified GC electrode was first voltammetrically scanned in an aqueous solution containing $5 \mathrm{M} \mathrm{NaClO}_{4}$ and $5 \mathrm{M}$ formate. No voltammetric signal was observed, as shown in Figure 1A (dotted line). Subsequently $0.96 \mu \mathrm{g}$ of N-CNT-Pd was drop-cast onto the GC electrode and left to dry under an $\mathrm{N}_{2}$ atmosphere. The amount of N-CNT-Pd drop-cast in the experiment corresponds to a coverage of $\sim 1$ monolayer, assuming the CNTs are perfectly tesselated on the electrode surface (see the Supporting Information). In reality a tangled, irregular multilayer is likely formed. Figure $1 \mathrm{~A}$ shows the $\mathrm{CV}$ of the above modified GC electrode in $5 \mathrm{M} \mathrm{NaClO}_{4}$ in the absence (dashed line) and presence (solid line) of $5 \mathrm{M}$ formate, respectively. The $\mathrm{CV}$ of the modified GC electrode in the formate solution shows a clear formate oxidation peak at -0.08 $\mathrm{V}$ vs SCE and a back anodic peak at around $-0.02 \mathrm{~V}$ vs SCE (solid line). No oxidation feature was observed in the absence of formate (dashed line). The formate oxidation is known to be highly surface sensitive, and at high overpotentials it is inhibited by the formation of a palladium oxide layer. ${ }^{6,11}$ It has been suggested that on the reverse scan the reduction of the oxide layer leads to the recommencement of the electrochemical process. $^{11}$

In order to characterize the palladium oxidation process, the voltammetric response of a bulk palladium microelectrode (radius $5.4 \mu \mathrm{m}$ ) was recorded in the absence of formate. The CV (black line in Figure S1A in the Supporting Information) in $5 \mathrm{M} \mathrm{NaClO}_{4}$ shows a pair of asymmetric redox peaks for Pd oxide formation and reduction: a broad Pd oxidation wave on the forward scan and a sharp peak for Pd oxide reduction on the backward scan. The reduction of the Pd oxide is found to occur at $\sim 0.0 \mathrm{~V}$ vs SCE. This potential is comparable to the peak potential of formate oxidation $(-0.08 \mathrm{~V}$ vs SCE) as recorded on the drop-cast N-CNT-Pd (solid line in Figure 1A). On the reverse scan of the voltammetry of formate oxidation at a drop-cast N-CNT-Pd electrode, a sharp increase of the oxidation current is observed (solid line in Figure 1A). This sharp increase in current is found to occur at the potentials of Pd oxide reduction where Pd active sites are released. ${ }^{11}$ This observation further evidences that the decrease of the oxidation current on the forward scan is caused, at least in part, by the formation of a Pd oxide layer.

We next consider the oxidation of methanol at a N-CNT-Pd modified GC electrode and at the $\mathrm{Pd}$ microelectrode. Voltammograms are shown in Figure $1 \mathrm{~B}$ and Figure S1B in the Supporting Information, respectively. The results for methanol oxidation mirror those found for formate. An anodic peak was observed at $-0.18 \mathrm{~V}$ vs SCE on the modified GC electrode in $1 \mathrm{M} \mathrm{NaOH}$ containing $10 \mathrm{M}$ methanol (solid line in Figure 1B), and a small oxidation peak was found on the backward scan at around $-0.45 \mathrm{~V}$ vs SCE. In the absence of methanol, the voltammetric responses of both the drop-cast $\mathrm{N}$ CNT-Pd (dashed line in Figure 1B) and the Pd microelectrode (black line in Figure S1B) demonstrate that Pd oxide reduction occurs near $-0.4 \mathrm{~V}$ vs SCE in $0.1 \mathrm{M} \mathrm{NaOH}$. This value of -0.4 $\mathrm{V}$ is more negative than the peak potential for methanol oxidation $(-0.18 \mathrm{~V}$ vs SCE) on the drop-cast N-CNT-Pd (solid line in Figure 1B). Hence, a significant surface coverage of $\mathrm{Pd}$ oxide has probably formed before the methanol oxidation peak current, and a further deactivation of the Pd active sites leads to significant inhibition toward methanol oxidation. ${ }^{10}$ Moreover, the methanol oxidation is significantly less favorable at the relatively negative potentials for Pd oxide reduction; hence, a much smaller back anodic peak (solid line in Figure 1B) is observed in comparison to that found with the formate oxidation (solid line in Figure 1A). The measured back-peaks in the two cases are independent of the potential limit (beyond a minimum threshold value). It should be noted that the increased current at high potentials $(>0.3 \mathrm{~V}$ vs SCE) in Figure 1B (solid line) corresponds to further methanol oxidation on the background carbon of the nanotube and also the oxidation of the solvent.

Formate oxidation on palladium is reported to result in the formation of carbon dioxide via a direct two-electron-transfer pathway, ${ }^{7,8}$ while methanol oxidation is reported to undergo a six-electron-transfer process at low overpotentials. ${ }^{32}$ For a microdisk electrode, the equation for the expected diffusion limited current is ${ }^{33}$

$$
I_{\lim }=4 n F D C r_{\mathrm{e}}
$$

where $n$ is the number of electrons transferred, $F$ is the Faraday constant $\left(96485 \mathrm{C} \mathrm{mol}^{-1}\right), D$ is the diffusion coefficient $(6.5 \times$ $10^{-11} \mathrm{~m}^{2} \mathrm{~s}^{-1}$ for formate ${ }^{34}$ and $4 \times 10^{-10} \mathrm{~m}^{2} \mathrm{~s}^{-1}$ for methanol $\left.{ }^{35}\right), C$ is the concentration of formate or methanol, and $r_{\mathrm{e}}$ is the electrode radius. Therefore, for $0.5 \mathrm{M}$ formate or methanol, the theoretical diffusion-limited currents at a $\mathrm{Pd}$ microelectrode with radius of $5.4 \mu \mathrm{m}$ can be estimated as $1.4 \times$ $10^{-7}$ and $2.5 \times 10^{-6} \mathrm{~A}$, respectively. However, the experimentally measured oxidative peak currents of $0.5 \mathrm{M}$ formate $\left(2.2 \times 10^{-9} \mathrm{~A}\right)$ and $0.5 \mathrm{M}$ methanol $\left(2.6 \times 10^{-8} \mathrm{~A}\right)$ at a $\mathrm{Pd}$ microelectrode $(r=5.4 \mu \mathrm{m})$ (red lines in Figure S1 in the Supporting Information) are much smaller than the hypothetical diffusion-limited values, evidencing that both the formate and methanol oxidation on Pd are surface reaction rate limited processes, rather than transport controlled.

It is worth mentioning that the modified GC electrode showed a slightly higher peak current of formate oxidation (8.3 $\left.\times 10^{-5} \mathrm{~A}\right)$ in the solution without the supporting electrolyte $\mathrm{NaClO}_{4}$ (see Figure S2 in the Supporting Information), indicating that the extremely high concentration of formate used is able to be self-supporting during the measurement, and suggests that some $\mathrm{Pd}$ active sites may be blocked by $\mathrm{ClO}_{4}{ }^{2-}$. Aqueous $5 \mathrm{M}$ formate solution was used in the following experiments.

3.2. CV of a Carbon Microwire Electrode in Situ Modified with a Dilute Coverage of Individual N-CNTPds. The previous section reported the voltammetric response of a multilayered, porous drop-cast surface. The interpretation of such a layer is complex and is hard to analyze. ${ }^{13-16}$ In order to study the formate oxidation on adsorbed but spatially well 

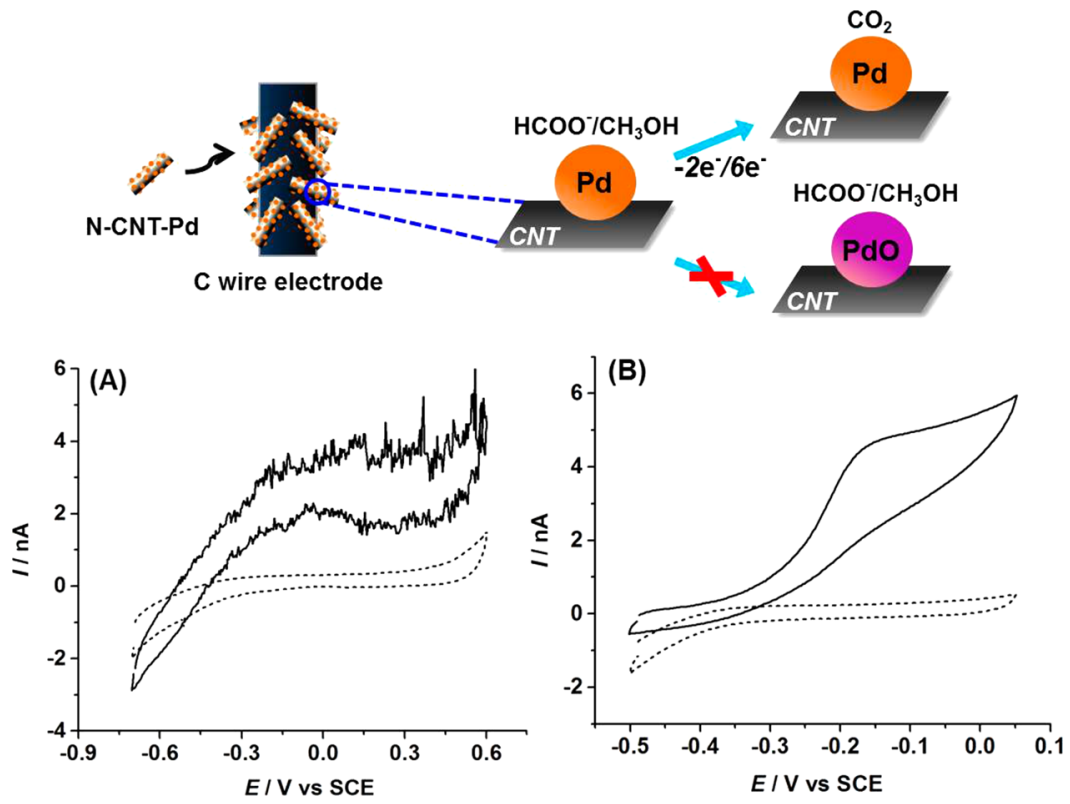

Figure 2. (A) CV recorded immediately after a carbon microwire electrode $(l \approx 1 \mathrm{~mm} ; d \approx 7 \mu \mathrm{m})$ was inserted in a $0.65 \mathrm{pM}$ suspesion of N-CNTPd containing $5 \mathrm{M}$ formate (dashed line) and after the electrode was in situ modified in the same solution for $30 \mathrm{~min}$ (solid line). Scan rate: $50 \mathrm{mV}$ $\mathrm{s}^{-1}$. (B) CV recorded immediately after a carbon microwire electrode was inserted in a $0.65 \mathrm{pM}$ suspesion of N-CNT-Pd containing $1 \mathrm{M} \mathrm{NaOH}+$ $10 \mathrm{M}$ methanol (dashed line) and after the electrode was in situ modified in the same solution for $60 \mathrm{~min}$ (solid line). Scan rate: $50 \mathrm{mV} \mathrm{s}{ }^{-1}$.

separated individual N-CNT-Pds, a carbon microwire electrode $(l \approx 1 \mathrm{~mm} ; d \approx 7 \mu \mathrm{m})$ was first treated with an in situ modification method by dipping the wire electrode in a suspension of N-CNT-Pd (0.65 pM) containing formate for 0.5 $\mathrm{h}$, followed by a CV sweep. During the modification process, about 100 individual N-CNT-Pds (see the Supporting Information) collided with and became immobilized on the microwire electrode, giving an electrode modified with enough $\mathrm{N}$-CNT-Pd to give a clear formate oxidation signal in CV. For the wire electrode used, one monolayer of N-CNT-Pds, assuming the CNTs are perfectly tessellted on the electrode surface, would be $3.4 \times 10^{4} \mathrm{CNTs}$ (see the Supporting Information). Therefore, only $0.3 \%$ of the surface area is covered by $100 \mathrm{~N}-\mathrm{CNT}-\mathrm{Pds}$, which gives large spatial separation of N-CNT-Pds on the wire electrode surface. No voltammetric response was detected when $\mathrm{CV}$ was recorded immediately after the wire electrode was dipped in the suspension of N-CNT-Pd (dashed lines in Figure 2A). After in situ modification in the formate solution dispersed with $\mathrm{N}$ CNT-Pd for $0.5 \mathrm{~h}$, the wire electrode showed a Faradaic current resulting from formate oxidation in the $\mathrm{CV}$ (solid lines in Figure 2A). Similar experiments were conducted to study methanol oxidation in $1 \mathrm{M} \mathrm{NaOH}$ containing $10 \mathrm{M}$ methanol. It was observed that a methanol oxidation signal was also shown in the $\mathrm{CV}$ at the wire electrode after $1 \mathrm{~h}$ of in situ modification in a suspension of N-CNT-Pd containing methanol (solid lines in Figure 2B).

In sharp contrast to the clear oxidation peaks shown in the $\mathrm{CV}$ of the drop-cast experiments, the wire electrode after in situ modification with N-CNT-Pd exhibits steady state currents at high overpotentials for both formate and methanol oxidation, indicating that neither process is inhibited by $\mathrm{Pd}$ oxide formation under these experimental conditions! Note that, for the $\mathrm{CV}$ of the N-CNT-Pd modified wire electrode, current fluctuations, as shown in Figure 2, are variable due to the different states of absorbed carbon nanotubes on the wire electrode surface. Such Faradaic current fluctuations have been explained in detail in a recent paper. ${ }^{36}$

3.3. Nanoimpacts of $\mathrm{N}-\mathrm{CNT}-\mathrm{Pd}$. Nanoimpact experiments of N-CNT-Pd were performed with the aim of separately studying the formate and methanol oxidation on individual impacting single N-CNT-Pd. To achieve this, a clean carbon microwire electrode was first inserted in a suspension of $\mathrm{N}$ CNT-Pd (0.65 pM) containing $5 \mathrm{M}$ formate. Chronoamperograms were then recorded at a series of applied potentials from -0.5 to $+0.25 \mathrm{~V}$ vs SCE. Figure $3 \mathrm{~A}$ shows a typical chronoamperogram at $+0.2 \mathrm{~V}$ vs SCE where oxidative spikes are observed, evidencing the impact of the individual carbon nanotubes. No spikes were detected at this potential in the absence of N-CNT-Pd (Figure S3 in the Supporting Information). A total number of 135 current spikes at $+0.2 \mathrm{~V}$ vs SCE were recorded, giving an average current of $(4.1 \pm 1.5)$ $\times 10^{-11} \mathrm{~A}$, and their height distribution is shown in Figure 3B. The average duration of the spike is short, about $30 \mathrm{~ms}$. This short duration is possibly caused by the formation of $\mathrm{CO}_{2}$ gas bubbles on the palladium surface ${ }^{37}$ or due to adsorbed carbon intermediates, $\mathrm{CO}_{\mathrm{ad}}$, produced by the further reduction of $\mathrm{CO}_{2}{ }^{38}$ leading to the deactivation of Pd active sites. The mean currents of the individual spikes as a function of potential from nanoimpacts of N-CNT-Pd are shown in Figure 3C, where it is observed that the average current at potentials more positive than $-0.2 \mathrm{~V}$ vs SCE shows a plateau at around $40 \mathrm{pA}$. No spikes were detected at potentials more negative than $-0.4 \mathrm{~V}$ vs SCE (Figure S4 in the Supporting Information). The average current from the spikes is found to have an onset at the potentials at which formate oxidation is observed in the $\mathrm{CV}$ (Figure 1A), evidencing that the detected current spikes correspond to Faradaic currents associated with formate oxidation catalyzed by the individual impacting N-CNT-Pd.

By modeling the N-CNT-Pd as a cylindrical electrode $(r=$ $\left.6.5 \times 10^{-8} \mathrm{~m} ; l=5 \times 10^{-6} \mathrm{~m}\right)$, for a mass transport controlled process, the expected peak current on single N-CNT-Pd from the oxidation of formate $(5 \mathrm{M})$ can be estimated as $3.68 \times 10^{-7}$ 

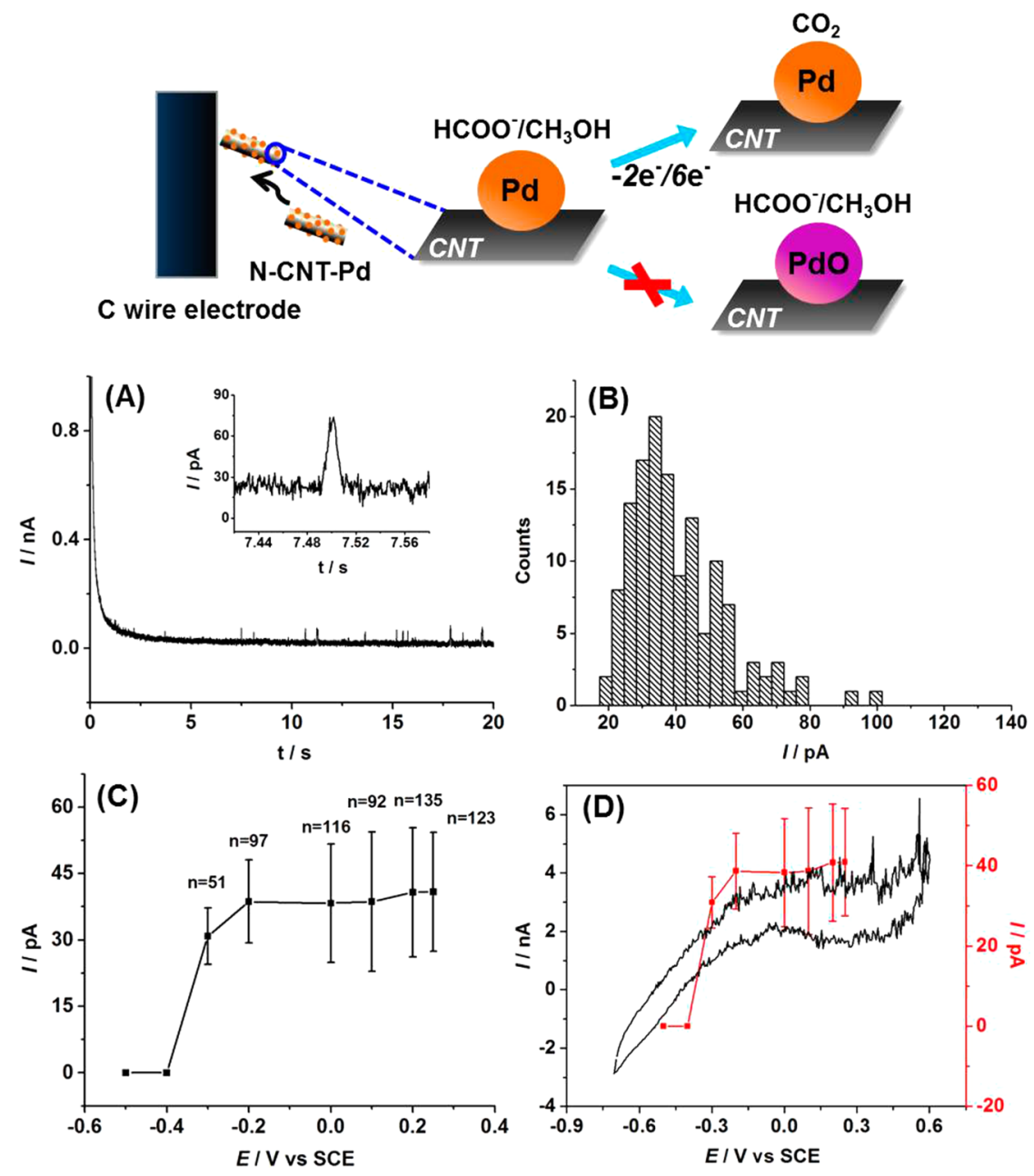

Figure 3. (A) Typical chronoamperogram obtained at $0.2 \mathrm{~V}$ vs SCE for a carbon microwire electrode in a $0.65 \mathrm{pM}$ suspesion of N-CNT-Pd containing $5 \mathrm{M}$ formate. (B) Height distribution of 135 current spikes at $0.2 \mathrm{~V}$ vs SCE. (C) Squares denoting the mean current of individual spikes at different potentials from nanoimapcts of N-CNT-Pd, where $n$ is the number of spikes. (D) Comparison of cyclic voltammetry of formate oxidation at the wire electrode in situ modified with well-separated N-CNT-Pd (black line) and nanoimpact analysis (red line) of formate oxidation on individual impacting N-CNT-Pd.

A. The average current of the spikes from formate oxidation is about $40 \mathrm{pA}$, which is significantly smaller than the expected peak current $\left(3.68 \times 10^{-7} \mathrm{~A}\right)$ for a mass transport control process. This further evidences that formate oxidation on individual N-CNT-Pds is a surface reaction rate limited process, rather than being under mass transport control. Moreover, from the drop-casting experiments, the expected current of formate oxidation at a single N-CNT-Pd (ca. $5 \mu \mathrm{m}$ in length, $130 \mathrm{~nm}$ in diameter) can be estimated as $7.9 \mathrm{pA}$ by directly dividing the peak current $\left(8.3 \times 10^{-5} \mathrm{~A}\right)$ (Figure S2 in the Supporting Information) by the amount of drop-cast N-CNT-Pds $\left(1.0_{5} \times\right.$ $10^{7}$ ) (see the Supporting Information). This value of $7.9 \mathrm{pA}$ is much less than the average current $(\sim 40 \mathrm{pA})$ detected from the nanoimpact experiment. Therefore, the higher currents likely result from the physical isolation of the carbon nanotubes in the impact experiment; in contrast, the drop-cast material will inevitably form irregular aggregated multilayers on the GC electrode surface.

In the nanoimpact experiments, the collision of individual $\mathrm{N}$ CNT-Pd with the wire electrode creates extremely high mass transport conditions for formate oxidation because of the small physical size of the individual carbon nanotubes. ${ }^{28,29}$ Nanoimpact analysis shows that the average current from formate oxidation on individual N-CNT-Pd remains stable even at the high overpotentials. The stable oxidation current is in agreement with the steady state current observed in the in situ modification experiments (Figure 3D), further evidencing that under high mass transport conditions $\mathrm{Pd}$ oxide formation is significantly inhibited, and as a result higher electro-oxidation efficiency for formate was achieved.

Nanoimpact experiments were also performed to detect the signals of methanol oxidation on individual N-CNT-Pds, but no spikes were detected (Figure S5 in the Supporting Information). On the basis of the drop-casting expeiments, the expected current from methanol oxidation on single $\mathrm{N}$ CNT-Pd is calculated to be $1.3 \mathrm{pA}$. Although the average current from individual impacting N-CNT-Pds might be several times larger than $1.3 \mathrm{pA}$ in the nanoimpact experiments according to the results of formate oxidation, it is still too small to be detected due to the comparable background noise $(\sim 20$ pA) (Figure S5).

3.4. Comparison and Inferences. We next compare the CVs of formate oxidation and methanol oxidation on the GC electrode drop-cast with N-CNT-Pd and their CVs at the wire electrode modified with a dilute coverage of well separated $\mathrm{N}$ CNT-Pd. A striking difference is that a steady state current is 

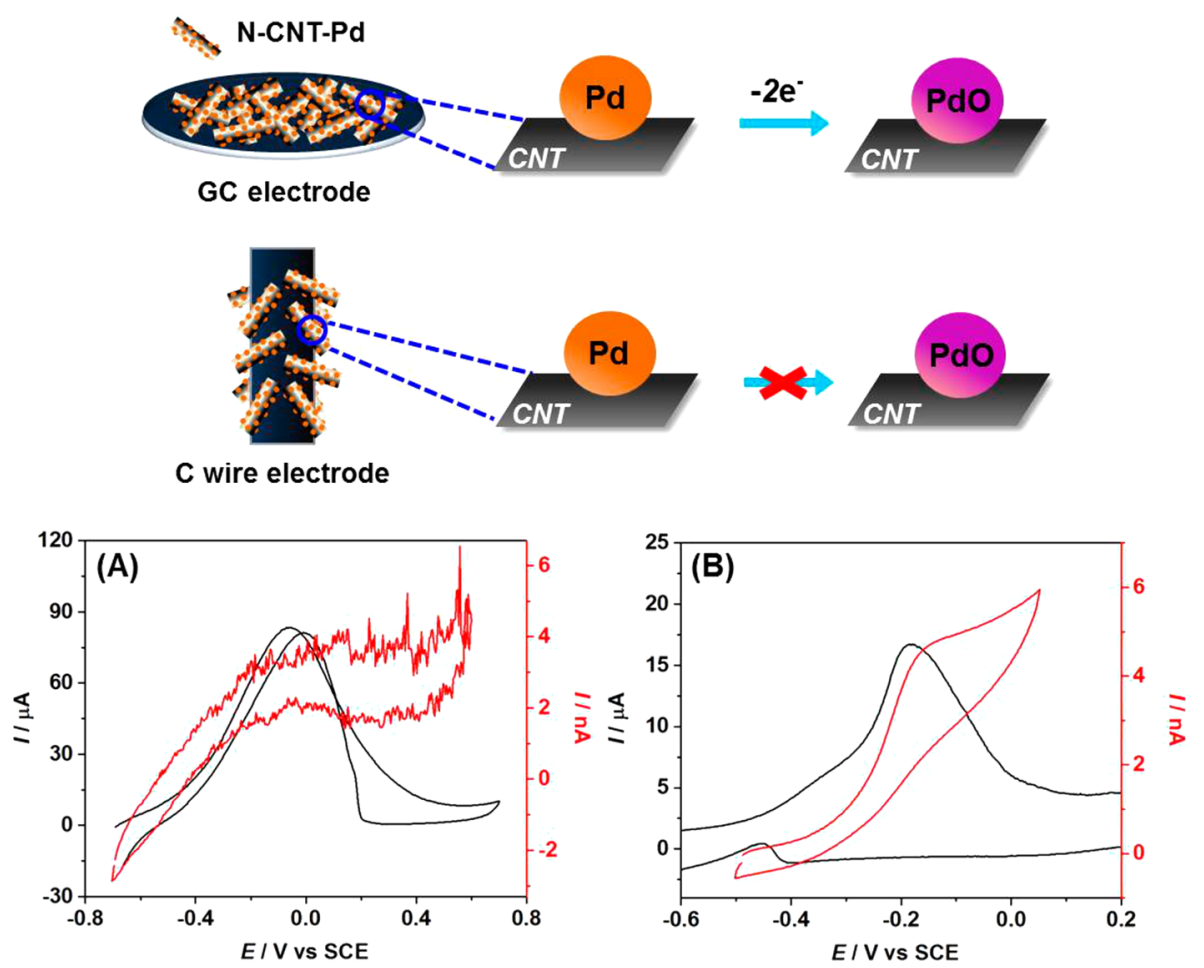

Figure 4. Comparison of cyclic voltammograms of formate oxidation (A) and methanol oxidation (B) on an N-CNT-Pd drop-cast GC electrode (black lines) and a carbon microwire electrode in situ modified with well-separated N-CNT-Pd (red lines).

observed in both formate and methanol oxidation at spatially well separated individual N-CNT-Pd, instead of the current peaks as shown in the CV response of the drop-cast N-CNT-Pd (Figure 4). Consequently, it is concluded that, for wellseparated individual N-CNT-Pd, the palladium oxidation (eq 3) as a competitive reaction is significantly inhibited; hence, the majority of $\mathrm{Pd}$ active sites on palladium nanoparticles remain active even at highly oxidizing potentials. The Pd active sites therefore continue to contribute to the catalysis of the formate or methanol oxidation at high potentials (eqs 1 and 2):

$$
\begin{aligned}
& \mathrm{HCOO}^{-} \rightarrow \mathrm{CO}_{2}+\mathrm{H}^{+}+2 \mathrm{e}^{-} \\
& \mathrm{CH}_{3} \mathrm{OH}+\mathrm{H}_{2} \mathrm{O} \rightarrow \mathrm{CO}_{2}+6 \mathrm{H}^{+}+6 \mathrm{e}^{-} \\
& \mathrm{Pd}+\mathrm{H}_{2} \mathrm{O} \rightarrow \mathrm{PdO}+2 \mathrm{H}^{+}+2 \mathrm{e}^{-}
\end{aligned}
$$

It was reported previously that palladium oxide formation was retarded in the presence of formic acid or methanol. ${ }^{10,39}$ Thus, a likely factor responsible for the observed steady state current is that well-separated individual N-CNT-Pds absorbed on the wire electrode surface experience very high mass transport conditions for formate or methanol oxidation. This provides a basis for the preference of formate or methanol oxidation during the competitive processes with palladium oxidation. In contrast, an intermingled porous "mat" of carbon nanotubes on the GC electrode is inevitably formed when using the drop-cast technique. The resulting relatively limited mass transport conditions for the oxidation of formate or methanol facilitate the formation of the $\mathrm{Pd}$ oxide layer; probably the mass transport is a mixture of linear semi-infinite diffusion and thinlayer transport. For well-separated individual N-CNT-Pds, there are large numbers of redox species around which create high mass transport conditions, while an overlapping diffusion regime is formed on the "mat" of N-CNT-Pds due to the confined volume of the available redox species. ${ }^{17-19,40,41}$ CVs similar to those seen with the drop-cast N-CNT-Pd were observed when the carbon microwire electrode was inserted in a suspension of N-CNT-Pds of an extremely high concentration $(9.2 \mathrm{pM})$ (10 times higher than that used in the in situ modification method) for about $10 \mathrm{~s}$, creating a higher population of large N-CNT-Pd ensembles which stick to the wire electrode surface and are then transferred into the solution containing formate or methanol to run a CV. As shown in Figure $S 6$ in the Supporting Information, the oxidation current falls off at high overpotentials in the CV of both formate and methanol oxidation, further evidencing that current inhibition by Pd oxide formation occurs on the large ensembles of the $\mathrm{N}$ CNT-Pd. It is also demonstrated that the voltammetric response of the N-CNT-Pd toward formate or methanol oxidation can be easily tailored by using different methods to modify the electrodes.

Overall, Pd oxide formation and formate or methanol oxidation are two competitive processes. The inferior efficiency of formate and methanol oxidation on Pd in their application to fuel cells is due in part to the significant inhibition of Pd oxide formation toward these oxidation processes. From the above comparison, it is seen that $\mathrm{Pd}$ oxide formation as a competitive reaction can be retarded under high mass transport conditions for formate and methanol oxidation, which in this work was created on the well-separated catalyst N-CNT-Pd. This highlights how the design of the catalyst in fuel cells and the local mass transport conditions play an important role in the energy conversion performance.

\section{CONCLUSIONS}

In this work, we recorded and compared the voltammograms of formate/methanol oxidation on drop-cast N-CNT-Pd and on wire electrodes modified with well-separated individual $\mathrm{N}$ - 
CNT-Pd. It was found that the inhibition from the Pd oxide formation on the oxidative processes on the drop-cast N-CNT$\mathrm{Pd}$ became retarded with the use of well-separated individual N-CNT-Pd, where high mass transport conditions prevail. Further evidence was seen in the study of formate oxidation on a single N-CNT-Pd via the nanoimpact method, in which the oxidation process was also not hindered by Pd oxidation at high overpotentials. The discovery of the superior electrocatalytic performance under high mass transport conditions suggests requirements for the design of fuel cell development and offers the scope for new catalytic approaches to be developed.

\section{ASSOCIATED CONTENT}

\section{S Supporting Information}

The Supporting Information is available free of charge on the ACS Publications website at DOI: 10.1021/acscatal.6b02023.

Figures S1-S6 as described in the text, estimation of the number of N-CNT-Pds in 1 monolayer on the GC electrode surface and the wire electrode surface, estimation of the number of N-CNT-Pds drop-cast on the GC electrode, and estimation of the amount of $\mathrm{N}$ CNT-Pds adsorbed on the wire electrode with in situ modification method (PDF)

\section{AUTHOR INFORMATION}

\section{Corresponding Author}

*E-mail for R.G.C.: richard.compton@chem.ox.ac.uk.

\section{Author Contributions}

$\S$ These authors contributed equally.

\section{Notes}

The authors declare no competing financial interest.

\section{ACKNOWLEDGMENTS}

This research was supported by the European Research Council (ERC) under the European Union's Seventh Framework Programme (FP/2007-2013), ERC Grant Agreement no. 320403. The China Scholarship Council is gratefully acknowledged for funding the Ph.D. of X.L. L.S. thanks the National Natural Science Foundation of China (No. 21403137) for support.

\section{REFERENCES}

(1) Wasmus, S.; Küver, A. J. Electroanal. Chem. 1999, 461, 14-31.

(2) Hamnett, A. Catal. Today 1997, 38, 445-457.

(3) Rice, C.; Ha, S.; Masel, R. I.; Waszczuk, P.; Wieckowski, A.; Barnard, T. J. Power Sources 2002, 111, 83-89.

(4) Steele, B. C. H.; Heinzel, A. Nature 2001, 414, 345-352.

(5) Rees, N. V.; Compton, R. G. J. Solid State Electrochem. 2011, 15, 2095-2100.

(6) Chen, A.; Ostrom, C. Chem. Rev. 2015, 115, 11999-12044.

(7) Jiang, K.; Zhang, H. X.; Zou, S.; Cai, W. B. Phys. Chem. Chem. Phys. 2014, 16, 20360-20376.

(8) Adams, B. D.; Asmussen, R. M.; Ostrom, C. K.; Chen, A. J. Phys. Chem. C 2014, 118, 29903-29910.

(9) Bianchini, C.; Shen, P. K. Chem. Rev. 2009, 109, 4183-4206.

(10) Takamura, T.; Sato, Y. Electrochim. Acta 1974, 19, 63-68.

(11) Capon, A.; Parsons, R. J. Electroanal. Chem. Interfacial Electrochem. 1973, 44, 239-254.

(12) Pan, Y.; Zhang, R.; Blair, S. L. Electrochem. Solid-State Lett. 2009, 12, B23-B26.

(13) Ward, K. R.; Gara, M.; Lawrence, N. S.; Hartshorne, R. S.; Compton, R. G. J. Electroanal. Chem. 2013, 695, 1-9.
(14) Henstridge, M. C.; Dickinson, E. J. F.; Aslanoglu, M.; BatchelorMcAuley, C.; Compton, R. G. Sens. Actuators, B 2010, 145, 417-427.

(15) Streeter, I.; Wildgoose, G. G.; Shao, L.; Compton, R. G. Sens. Actuators, B 2008, 133, 462-466.

(16) Batchelor-McAuley, C.; Compton, R. G. J. Phys. Chem. C 2014, 118, 30034-30038.

(17) Toh, H. S.; Jurkschat, K.; Compton, R. G. Chem. - Eur. J. 2015, 21, 2998-3004.

(18) Cloake, S. J.; Toh, H. S.; Lee, P. T.; Salter, C.; Johnston, C.; Compton, R. G. ChemistryOpen 2015, 4, 22-26.

(19) Toh, H. S.; Batchelor-McAuley, C.; Tschulik, K.; Uhlemann, M.; Crossley, A.; Compton, R. G. Nanoscale 2013, 5, 4884-4893.

(20) Xiao, X.; Bard, A. J. J. Am. Chem. Soc. 2007, 129, 9610-9612.

(21) Bard, A. J.; Zhou, H.; Kwon, S. J. Isr. J. Chem. 2010, 50, $267-$ 276.

(22) Ly, L. S. Y.; Batchelor-McAuley, C.; Tschulik, K.; Kätelhön, E.; Compton, R. G. J. Phys. Chem. C 2014, 118, 17756-17763.

(23) Li, X.; Batchelor-McAuley, C.; Tschulik, K.; Shao, L.; Compton, R. G. ChemPhysChem 2015, 16, 2322-2325.

(24) Cheng, W.; Compton, R. G. Angew. Chem., Int. Ed. 2015, 54, $7082-7085$

(25) Pumera, M. ACS Nano 2014, 8, 7555-7558.

(26) Rees, N. V. Electrochem. Commun. 2014, 43, 83-86.

(27) Cheng, W.; Compton, R. G. TrAC, Trends Anal. Chem. 2014, 58, $79-89$.

(28) Li, X.; Lin, C.; Batchelor-McAuley, C.; Laborda, E.; Shao, L.; Compton, R. G. J. Phys. Chem. Lett. 2016, 7, 1554-1558.

(29) Li, X.; Batchelor-McAuley, C.; Whitby, S. A. I.; Tschulik, K.; Shao, L.; Compton, R. G. Angew. Chem., Int. Ed. 2016, 55, 4296-4299.

(30) Ellison, J.; Batchelor-McAuley, C.; Tschulik, K.; Compton, R. G. Sens. Actuators, B 2014, 200, 47-52.

(31) Ellison, J.; Tschulik, K.; Stuart, E. J. E.; Jurkschat, K.; Omanović, D.; Uhlemann, M.; Crossley, A.; Compton, R. G. ChemistryOpen 2013, $2,69-75$

(32) Takamura, T.; Mochimaru, F. J. Electrochem. Soc. 1967, 114, $1251-1254$

(33) Shoup, D.; Szabo, A. J. Electroanal. Chem. Interfacial Electrochem. 1982, 140, 237-245.

(34) Wang, Y.; Wu, B.; Gao, Y.; Tang, Y.; Lu, T.; Xing, W.; Liu, C. J. Power Sources 2009, 192, 372-375.

(35) Danaee, I.; Jafarian, M.; Mirzapoor, A.; Gobal, F.; Mahjani, M. G. Electrochim. Acta 2010, 55, 2093-2100.

(36) Hodson, H.; Li, X.; Batchelor-McAuley, C.; Shao, L.; Compton, R. G. J. Phys. Chem. C 2016, 120, 6281-6286.

(37) Mikołajczuk, A.; Borodzinski, A.; Kedzierzawski, P.; Stobinski, L.; Mierzwa, B.; Dziura, R. Appl. Surf. Sci. 2011, 257, 8211-8214.

(38) Wang, J. Y.; Zhang, H. X.; Jiang, K.; Cai, W. B. J. Am. Chem. Soc. 2011, 133, 14876-14879.

(39) Miyake, H.; Okada, T.; Samjeske, G.; Osawa, M. Phys. Chem. Chem. Phys. 2008, 10, 3662-3669.

(40) Gara, M.; Laborda, E.; Holdway, P.; Crossley, A.; Jones, C. J. V.; Compton, R. G. Phys. Chem. Chem. Phys. 2013, 15, 19487-19495.

(41) Schneider, A.; Colmenares, L.; Seidel, Y. E.; Jusys, Z.; Wickman, B.; Kasemo, B.; Behm, R. J. Phys. Chem. Chem. Phys. 2008, 10, 19311943. 